\title{
Estimation of the contact area of solids by electrothermal analogy
}

\author{
E. D. Eidelman ${ }^{1,2}$ \\ ${ }^{1}$ Ioffe Physico-Technical Institute of the Russian Academy of Sciences, \\ Polytechnicheskaya 26, Saint Petersburg, 194021, Russia \\ ${ }^{2}$ Saint Petersburg Chemical-Pharmaceutical Academy, Popova street 14, \\ Saint Petersburg, 197376, Russia \\ eidelman@mail.ioffe.ru
}

\section{DOI 10.17586/2220-8054-2015-6-4-547-550}

A method of thermal measurements has been proposed for determining the real surface contact area. Measurement of the true contact area is somewhat difficult. We propose here a method of contact area measurement, which is, in essence, an idealization of the well-known probe method employed in surface studies. In this study, to determine (estimate!) the fraction of the contact surface area projected onto the plane of a geometrical area of the section of the surface, it is proposed to use the electrothermal analogy. Considered in terms of this analogy, electrical conductance is assumed to correlate with heat transfer. As a result, the real contact area is found to be millions of times smaller than the area of the plane surface.

Keywords: tribology, contact area, Landauer's relation, electrothermal analogy.

Received: 29 April 2015 Revised: 5 May 2015

\section{Introduction}

Calculation of the true contact area between solid bodies, if it is to be made with due allowance for the processes involved, is an intricate problem of tribology, as well as that of engineering and materials sciences [1]. The space between the areas in contact is usually filled by an intermediate phase and products of destruction. This space is naturally pervaded by electric fields generated by atoms and molecules of the surface. Any attempt at modeling the real contact area should lean upon surface shape measurements made before contact [2], but the assumptions made in simulation about the shape of the overlaying contact surfaces largely depreciate these measurements [1].

The measurement of the true contact area is met with some difficulty. Indeed, measurements conducted by traditional methods involving contact with a painted surface will obviously yield an overestimate because of squeezing and adhesion, which, in the absence of a color layer, may be completely absent. The method based on measuring the electrical resistance of contacts does not actually permit operation without the application of pressure and the unavoidable fracture in the contact areas. The techniques involved in nondestructive testing depend markedly on the thickness of the layer within which radiation interacts with matter.

It is difficult to correctly estimate the effects of the potentials generated by surface atoms in these methods (for more details cf. [3, 4] and references therein).

We are proposing here a method of contact area measurement, which is, in essence, an idealization of the well-known probe method employed in surface studies. In this approach, the point probe is actually an atomically sized part of the surface of the body brought in contact with an atomically sized region of the body under study. 


\section{Formulation of the problem}

First, we let heat (phonons) and electric current (electrons) be transported only within small areas $S i(i=1,2,3 \ldots)$, within which the distance between atoms of the bodies brought in contact is on the order of the lattice constant of these bodies, or on the order of interatomic distances in amorphous bodies. In this study, to determine (estimate!) the fraction of the contact surface area $\sum_{i} S_{i \perp}=S_{r}$ projected onto the plane of a geometrical area $S$ of this section of the surface, we propose to use the electrothermal analogy.

Considered in terms of this analogy, electrical conductance $G$ is assumed to correlate with heat transfer, which in common notation (see, e.g., [5]), is written as $\alpha S_{r}$, where $\alpha$ is the heat transfer coefficient. The inverse quantity, $1 / \alpha$, is usually termed thermal resistance or the Kapitsa resistance. The use of the heat transfer coefficient at the boundaries of solids is fully justified. Indeed, contact between solids, except for very small areas, is actually that between the liquid mixtures covering these surfaces [5].

Let a certain amount of heat cross the boundary per unit time and unit area. Then, the heat conductance flux in the first body, which has the temperature $T_{1}$ far from the point of contact and $T_{1}^{\prime}$ at the boundary, the heat transfer flux between the boundaries with temperatures $T_{1}^{\prime}$ and $T_{1}^{\prime \prime}$, and, finally, the hear conductance flux in the second body with the temperatures $T_{2}^{\prime \prime}$ at the boundary and $T^{\prime \prime}$ far from it, are equal. These fluxes can be cast as

$$
\frac{\lambda_{1}}{\delta_{1}}\left(T_{1}-T_{1}^{\prime}\right)=\alpha\left(T_{1}^{\prime}-T_{2}^{\prime \prime}\right)=\frac{\lambda_{2}}{\delta_{2}}\left(T_{2}^{\prime \prime}-T^{\prime \prime}\right),
$$

where $\lambda_{j}$ and $\delta_{j}$ are, accordingly, the thermal conductivities and the characteristic dimensions of temperature variation for each of the bodies, with $j=1,2$. Obviously enough, at the boundary, there is a temperature jump $\delta T=T_{1}^{\prime}-T_{2}^{\prime \prime}$.

The main assumption maintained in this work is that a solid can be considered as an array of quasi-one-dimensional linear filaments. We will limit ourselves to a purely mechanical model of connection [6-7], neglecting all rheological effects, adhesion, structure change as a result of contact, and all electric and quantum potentials of interactions. This should not qualitatively change the estimates, to which we are going to adhere in what follows.

The conductivity of a quantum point contact between two one-dimensional conductors, one of which is located in body 1 , and the other, in body 2 , can be calculated in the quantum one-dimensional filament approximation using Landauer's relation [8, 9]:

$$
G=G_{0} \sum t_{i}\left(n_{i 1}-n_{i 2}\right)
$$

where $t_{i}$ is the normalized squared amplitude of transit of an electron with charge $e$ in contact numbered $i, n_{i j}$ is the number of such electrons specified in accordance with the Fermi-Dirac distribution for each of the conductors with the number $j=1,2, G_{0}=e^{2} /(\pi \hbar)$ is the conductance quantum, and, as usual, $\hbar$ is Planck's constant.

By electrothermal analogy, for the heat transfer quantum we have $\hbar \omega d \omega /(\pi \hbar \delta T)$, where $\omega$ is the phonon frequency, and $\delta T$ is the temperature jump at the boundary. The frequency $\omega$ must naturally be smaller than the lowest of the Debye frequencies $\omega^{*}$ characteristic of bodies in contact.

Thus, Eq. (1) for heat transfer can be recast as:

$$
\alpha S_{r}=\frac{1}{\pi} \int_{0}^{\omega_{*}} \hbar \omega d \omega \frac{n_{1}-n_{2}}{\delta T} t
$$


where $t$ is the normalized squared amplitude of transfer, i.e., the transfer coefficient of a phonon of frequency $\hbar \omega$, and

$$
n_{j}=\frac{1}{\exp \left(\frac{\hbar \omega}{k T_{j}}\right)-1}
$$

is the number of such phonons specified in accordance with the Bose-Einstein distribution for each of the contacting bodies numbered $j=1,2$, and $k$ is the Boltzmann constant.

\section{Estimation of the projection of contact area}

Obviously, at high enough temperatures $\left(n_{1}-n_{2}\right) / \delta T \cong k /(\hbar \omega)$.

The square of the amplitude $t$ of phonon transfer (vibration) over two semi-infinite one-dimensional chains coupled elastically at the boundary was calculated for frequencies lower than the lowest of the Debye frequencies for the corresponding contacting bodies [5]. It was shown [5] that $t$ may be considered frequency independent and equal to:

$$
t=\frac{4 \rho_{1} c_{1} \rho_{2} c_{2}}{\left(\rho_{1} c_{1}+\rho_{2} c_{2}\right)^{2}}
$$

where $\rho_{j}$ and $c_{j}$ are, accordingly, the densities and sound velocities in the bodies in contact.

In this approximation, the integral in Eq. (2) can readily be taken to yield:

$$
\alpha S_{r}=\frac{k^{2} \Theta_{*}}{\pi \hbar} \frac{4 a}{(1+a)^{2}}
$$

where $\Theta^{*}$ is the lowest of the Debye temperatures for the materials in contact, and the parameter

$$
a=\frac{\rho_{1}}{\rho_{2}} \frac{c_{1}}{c_{2}},
$$

is the relative characteristic of the contacting materials. It is usually a quantity on the order of unity.

In an experiment, one usually studies the amount of heat $P$ crossing a "geometric" surface per unit time. It is known that $P=\alpha S \delta T$. Thus, we come to the relation suitable for the subsequent estimation:

$$
\frac{S_{r}}{S}=\frac{k^{2} \Theta_{*}}{\pi \hbar} \frac{4 a}{(1+a)^{2}} \frac{\delta T}{P}
$$

The temperature jump at the boundary is usually [1] on the order of $\delta T \cong 10 K$, already at milliwatt-scale power, so that the real contact area turns out to be millions of times smaller than the area of the plane surface. Taking into due account the various rheological phenomena, adhesion and interaction potentials may significantly alter this estimate.

The relation operating with a temperature difference between the contacting bodies offset from the points of contact, $\Delta T=T^{\prime}-T^{\prime \prime}$, would probably be more suitable for practical measurements than Eq. (3). We thus come to the following equation:

$$
\frac{S_{r}}{S}=\frac{k^{2} \Theta_{*}}{\pi \hbar} \frac{4 a}{(1+a)^{2}}\left[\frac{\Delta T}{P}-\frac{1}{S}\left(\frac{\delta_{1}}{\lambda_{1}}+\frac{\delta_{2}}{\lambda_{2}}\right)\right] .
$$

\section{Conclusion}

A method of thermal measurement has been proposed for determining the real surface contact area. This method can be employed both directly for a preliminary evaluation of the contact area and in the method of mechanical or atomic force sensing to refine the contact area of the probe with a sample surface. 


\section{Acknowledgments}

The author expresses gratitude to A. P. Meilakhs for a discussion of Landauer's relation, as well as to S. A. Chizhik and M. L. Heifets for having made acquaintance with the state of the art of the problem. I am grateful to A. Ya. Vul' for interest in the work.

Support of the RAS Presidium programs "Fundamentals of nanostructure technologies and nanomaterial" and "Quantum mesoscopic and disordered systems", as well as of RFBR (Grants 14-08-90011 Bel-a) is gratefully acknowledged.

\section{References}

[1] Kaufman D. M. Coupled Principles for Computational Frictional Contact Mechanics. New Brunswick, New Jersey, 2009, 160p.

[2] Chizhik S.A., Wierzcholski K., Trushko A.V., Zhytkova M.A., Miszczak A. Properties of Cartilage on Micro- and Nanolevel. Advances in Tribology, 2010, 2010, Article ID 243150, 8 pages.

[3] Koval'chyk Yu. I., Shyrokov V. V., Modeling of Temperature Flashes under the Conditions of Periodic Contact of Friction Surfaces. Materials Science 3-2005, 41(2), P. 192-201.

[4] Koval'chyk Yu. I., Simulation of Wave Processes under Conditions of Friction of Rough Surfaces (Review). Materials Science, 2003, 39(4), P. 584-595.

[5] Faghri A., Zhang Y., Howell J., Advanced Heat and Mass Transfer. Columbia, MO: Global Digital Press., 2010, 550 p.

[6] Zhang U.L, Keblinski P., Wang J-S., Li B. L. Interfacial thermal transport in atomic junctions. Phys.Rev.B., 2011, 83, P. 064303-1-8.

[7] Meilakhs A. P., Eidelman E. D. New model of heat transport across the metal-insulator interface by the example of boundaries in a diamond-copper composite. JETP Letters, 2013, 97(1), P. 38-40.

[8] Landauer R. Spatial variation of currents and fields due to localized scatterers in metallic conduction. IBM Journal of Research and Development, 1957, 1, P. 223-231.

[9] Buttiker M., Imry Y., Landauer R., Pinhas S. Generalized-channel conductance formula with application to small rings. Phys. Rev. B., 1895, 31, P. 6207-6219. 